\title{
Identification of composite local linear state space models using a projected gradient search
}

\author{
Vincent Verdult, Lennart Ljung, and Michel Verhaegen \\ Department of Electrical Engineering \\ Linköping University, SE-581 83 Linköping, Sweden \\ WWW: http://www.control.isy.liu.se \\ Email: ljung@isy.liu.se
}

October 2, 2001

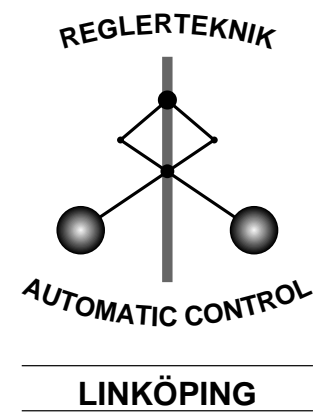

Report no.: LiTH-ISY-R-2359

Technical reports from the Automatic Control group in Linköping are available by anonymous ftp at the address ftp.control.isy.liu.se. This report is contained in the file 2359.pdf. 



\title{
Identification of composite local linear state space models using a projected gradient search
}

\author{
Vincent Verdult, Lennart Ljung, Michel Verhaegen
}

June 11, 2001

\begin{abstract}
An identification method is described to determine a weighted combination of local linear state space models from input and output data. Normalized radial basis function are used for the weights, and the system matrices of the local linear models are fully parameterized. By iteratively solving a nonlinear optimization problem, the centers and widths of the radial basis functions and the system matrices of the local models are determined. To deal with the nonuniqueness of the fully parameterized state space system, a projected gradient search algorithm is described. It is pointed out that when the weights depend only on the input, the dynamical gradient calculations in the identification method are stable. When the weights also depend on the output, certain difficulties might arise. The methods are illustrated using several examples that have been studied in the literature before.
\end{abstract}

\section{Introduction}

Local linear modeling is one of the many possibilities to approximate a nonlinear dynamical system. It is an attractive approach because the local models can be interpreted as linearizations of the nonlinear system in different operating points. By making a weighted combination of these linear models, one hopes to describe the complete nonlinear behavior sufficiently accurately (Murray-Smith and Johansen, 1997b). In this paper we want to determine a weighted combination of linear models from a finite number of measurements of the input and output of the system. This is the so-called black-box identification approach (Sjöberg et al., 1995). Black-box identification of local linear model structures has been studied mainly for inputoutput systems and for state space systems of which all states are measurable (Murray-Smith and Johansen, 1997b; Skeppstedt et al., 1992; Cao et al., 1997; Boukhris et al., 1999). The case where only part of the state is measurable is of course of more general interest. In this paper we discuss the identification of local linear state space models where the state is not measured directly, only a linear combination of some of the states is available as an output.

Why do we consider state space systems? Input-output models are in general easier to identify and can approximate a broad class of nonlinear systems. However, as argued by Rivals and Personnaz (1996) state space systems describe an even broader class of nonlinear systems. Although, it is always possible to convert a nonlinear input-output model into a state space model, the converse is not necessarily true, if the nonlinear mappings are required to be continuous and differentiable (Stark et al., 1997; Stark, 1999). A second observation made by Rivals and Personnaz (1996) is that even when an input-output representation of the system exists, state space systems are likely to require fewer parameters. In addition to 
these reasons, state space systems are attractive for dealing with multivariable inputs and outputs. Furthermore, many nonlinear control methods are based on state space systems.

Despite these advantages, the major drawback of state space systems is that they are recurrent (Nerrand et al., 1993). It is well-known that the identification of recurrent models can be troublesome (Haykin, 1999; Nerrand et al., 1994). In this paper we show that some of the problems associated with identification of recurrent networks can be overcome for the local linear model description by exploiting its special structure.

The paper is organized as follows. In Section 2 we discuss the model structure that we consider and discuss some of the problems related to the identification of such models. One of these problems, the non-uniqueness of the state space representation is dealt with in Section 3. Sections 4 and 5 are devoted to some special cases where the structure of the system can be exploited to facilitate the nonlinear optimization associated with the identification procedure. All these sections deal with the case of measurement noise at the output; in Section 6 we discuss the extension for a special kind of process noise. Finally, some numerical examples are presented in Section 7.

\section{Problem Description}

We consider systems of the form

$$
\begin{aligned}
x_{k+1} & =\sum_{i=1}^{s} p_{i}\left(\phi_{k}\right)\left(A_{i} x_{k}+B_{i} u_{k}+O_{i}\right) \\
y_{k} & =C x_{k}+v_{k},
\end{aligned}
$$

where $s$ is the number of local models, $x_{k} \in \mathbb{R}^{n}$ represents the unknown state, $u_{k} \in \mathbb{R}^{m}$ is the input, $y_{k} \in \mathbb{R}^{\ell}$ is the output, $p_{i}\left(\phi_{k}\right) \in \mathbb{R}$ is the weighting for the $i$ th model, and $v_{k} \in \mathbb{R}^{\ell}$ is an unknown white noise sequence that is independent of $u_{k}$. The weighting vectors $p_{k}$ depend on the scheduling vector $\phi_{k} \in \mathbb{R}^{q}$. This scheduling vector corresponds to the operating point of the system, typically it will depend on the input and the state, that is

$$
\phi_{k}=\psi\left(x_{k}, u_{k}\right)
$$

with $\psi: \mathbb{R}^{n m} \rightarrow \mathbb{R}^{q}$, which is assumed to be a known, possibly nonlinear function.

Depending on the number of local models used, the system (1)-(2) can be used to approximate the nonlinear system

$$
\begin{aligned}
x_{k+1} & =f\left(x_{k}, u_{k}\right) \\
y_{k} & =C x_{k}+v_{k},
\end{aligned}
$$

with $f$ a continuous and differentiable map, up to arbitrary accuracy (Johansen et al., 2000). An important problem is the determination of the number of local models required to obtain a specified accuracy. One possibility is to try different choices and use cross-validation. A similar approach can be used to determine the order of the state. However, the determination of these quantities is not addressed in this paper, and it is assumed that both the number of local models and the order of the state are known.

It is common practice in local linear modeling to use normalized radial basis functions for the weights $p_{i}\left(\phi_{k}\right)$. Let the $i$ th radial basis function be equal to

$$
r_{i}\left(\phi_{k} ; c_{i}, w_{i}\right)=\exp \left(-\left(\phi_{k}-c_{i}\right)^{T} \operatorname{diag}\left(w_{i}\right)^{2}\left(\phi_{k}-c_{i}\right)\right)
$$


then the $i$ th weight is taken as

$$
p_{i}\left(\phi_{k} ; c_{i}, w_{i}\right)=\frac{r_{i}\left(\phi_{k} ; c_{i}, w_{i}\right)}{\sum_{j=1}^{s} r_{j}\left(\phi_{k} ; c_{j}, w_{j}\right)} .
$$

The normalization ensures that the weights are always between zero and one, and that they sum up to one at every time instant. Normalization is common practice in local linear modeling, although some subtle problems can arise as discussed by Shorten and MurraySmith (1997).

The goal is to determine from a finite number of measurements of the input $u_{k}$ and output $y_{k}$, the local models $A_{i}, B_{i}, O_{i}$ and $C$; and the centers $c_{i}$ and weights $w_{i}$ of the radial basis functions. To determine these parameters, we propose to minimize the following cost function

$$
V_{N}(\theta, c, w):=\sum_{i=1}^{N}\left\|y_{k}-\hat{y}_{k}(\theta, c, w)\right\|_{2}^{2}=E_{N}(\theta, c, w)^{T} E_{N}(\theta, c, w),
$$

where

$$
E_{N}(\theta, c, w)^{T}=\left[\begin{array}{lll}
\left.\left(y_{1}-\hat{y}_{1}(\theta, c, w)\right)^{T} \quad\left(y_{2}-\hat{y}_{2}(\theta, c, w)\right)^{T} \quad \cdots \quad\left(y_{N}-\hat{y}_{N}(\theta, c, w)\right)^{T}\right]
\end{array}\right.
$$

the vectors $c$ and $w$ consist of the centers $c_{i}$ and $w_{i}$, respectively; $\theta$ is a set of parameters that describe the system matrices of the local models $A_{i}, B_{i}, O_{i}$ and $C$; and $\hat{y}_{k}(\theta, c, w)$, is the output of the model

$$
\begin{aligned}
x_{k+1}(\theta, c, w) & =\sum_{i=1}^{s} p_{i}\left(\phi_{k} ; c, w\right)\left(A_{i}(\theta) x_{k}(\theta, c, w)+B_{i}(\theta) u_{k}+O_{i}(\theta)\right) \\
\hat{y}_{k}(\theta, c, w) & =C(\theta) x_{k}(\theta, c, w) .
\end{aligned}
$$

The minimization of $V_{N}$ is a nonlinear, nonconvex problem because of the complicated dependence of $V_{N}$ on the parameters $\theta, c$, and $w$. In the neural network literature, solving this optimization problem is referred to as training the model. A commonly used technique to train recurrent networks is the Levenberg-Marquardt method, which is an iterative procedure that updates the parameters as follows:

$$
\left[\begin{array}{c}
\theta^{(i+1)} \\
c^{(i+1)} \\
w^{(i+1)}
\end{array}\right]=\left[\begin{array}{c}
\theta^{(i)} \\
c^{(i)} \\
w^{(i)}
\end{array}\right]-\mu^{(i)} H_{N}\left(\lambda^{(i)}, \theta^{(i)}, c^{(i)}, w^{(i)}\right)^{-1} \Psi_{N}^{T}\left(\theta^{(i)}, c^{(i)}, w^{(i)}\right) E_{N}\left(\theta^{(i)}, c^{(i)}, w^{(i)}\right)
$$

where $\mu^{(i)}$ is the step size,

$$
H_{N}(\lambda, \theta, c, w):=\lambda I+\Psi_{N}^{T}(\theta, c, w) \Psi_{N}(\theta, c, w)
$$

is the approximate Hessian, $\lambda^{(i)}$ is the Levenberg-Marquardt regularization parameter and

$$
\Psi_{N}(\theta, c, w):=\left[\begin{array}{lll}
\frac{\partial E_{N}(\theta, c, w)}{\partial \theta^{T}} & \frac{\partial E_{N}(\theta, c, w)}{\partial c^{T}} & \frac{\partial E_{N}(\theta, c, w)}{\partial w^{T}}
\end{array}\right]
$$

is the gradient. 
The model (3)-(4) can be viewed as a recurrent state space neural network (Haykin, 1999; Narendra and Parthasarathy, 1990) with a special structure. Since recurrent networks are difficult to train, special structures have been suggested that can be exploited to facilitate training. An example of this is the radial basis function network proposed by Gan and Danai (2000). The model that we consider in this paper, is another attempt to exploit special structure.

Training of our recurrent model (3)-(4) is difficult, because of three main reasons.

First, the state-space representation is not unique; there exist different state-space models that have the same input-output behavior. For the model we consider, it is readily seen that the same input output behavior is obtained by taking $\widetilde{x}_{k}=T^{-1} x_{k}, \widetilde{A}_{i}=T^{-1} A_{i} T$, $\widetilde{B}_{i}=T^{-1} B_{i}, \widetilde{O}_{i}=T^{-1} O_{i}$ and $\widetilde{C}=C T$. One way to deal with this nonuniqueness is to use an appropriate parameterization that makes the state-space representation unique. However, such representations may have bad numerical properties; therefore, in Section 3, we propose to use fully parameterized system matrices and we describe a method to deal with the similarity transformation during training. This method determines the directions in the parameter space that do not change the cost function, and does not update the parameters in this direction by using an appropriate projection for the gradient in (5). The method is based on ideas used for identification of linear and linear parameter-varying state space systems by McKelvey and Helmersson (1997), Lee and Poolla (1999), and Verdult and Verhaegen (2000).

The second reason is the complicated dependence of the gradient on the past data. It is easy to see from (3)-(4) that the gradient at time instant $k$ with respect to $\theta, c$ and $w$ depends on the entire past of the state sequence $x_{k}$. This dynamic dependence might lead to stability problems during training. We show in Sections 4 and 5 that for certain special choices of the scheduling vector $\phi_{k}$, attractive training methods exist.

If the model is used to approximate a nonlinear system, there is a third reason that makes training difficult. The original system can be approximated by different models of the type (3)-(4), equally well. For the model we consider, a simple example is to shift the operating points. This problem has been discussed by Murray-Smith and Johansen (1997a) and Shorten et al. (1999). Remedies are local learning (Murray-Smith and Johansen, 1997a; Bottou and Vapnik, 1992), regularization and early stopping (Haykin, 1999; Sjöberg and Ljung, 1995; Johansen, 1997). Although we do not discuss these techniques in the paper, they can all be applied for training the model structure we consider.

\section{Identification Based on Projected Gradient Search}

For easy of notation, we introduce the following matrices:

$$
\begin{aligned}
\bar{A} & :=\left[\begin{array}{llll}
A_{1} & A_{2} & \ldots & A_{s}
\end{array}\right] \\
\bar{B} & :=\left[\begin{array}{llll}
B_{1} & B_{2} & \ldots & B_{s}
\end{array}\right] \\
\bar{O} & :=\left[\begin{array}{llll}
O_{1} & O_{2} & \ldots & O_{s}
\end{array}\right] \\
\bar{C} & :=\left[\begin{array}{llll}
C & 0 & \ldots & 0
\end{array}\right]
\end{aligned}
$$

We also define

$$
\Theta:=\left[\begin{array}{ccc}
\bar{A} & \bar{B} & \bar{O} \\
\bar{C} & 0 & 0
\end{array}\right]
$$


This matrix is used to define the parameters $\theta$ that describe the local linear models as

$$
\theta:=\Pi \operatorname{vec}(\Theta)
$$

where $\Pi$ is a selection matrix such that the entries of $\Theta$ that are zero by definition do not appear in $\theta$. Since $\theta$ contains all the entries of the matrices $\bar{A}, \bar{B}, \bar{O}$ and $C$, it is clear that we have fully parametrized the system matrices that describe the local linear models. With this approach it is important to deal with similarity transformations of the state in an appropriate way. Since such transformations do not change the input-output behavior of the system, they can cause trouble with the update rule (5).

Similar to Lee and Poolla (1999) we define the similarity map $S_{\Theta}: T \in \mathbb{R}^{n \times n}, \operatorname{det}(T) \neq 0 \rightarrow$ $\mathbb{R}^{(n+\ell) \times(n+m+1) s}$ as

$$
S_{\Theta}(T):=\left[\begin{array}{cc}
T^{-1} & 0 \\
0 & I_{\ell}
\end{array}\right]\left[\begin{array}{ccc}
\bar{A} & \bar{B} & \bar{O} \\
\bar{C} & 0 & 0
\end{array}\right]\left[\begin{array}{ccc}
T_{s} & 0 & 0 \\
0 & I_{m s} & 0 \\
0 & 0 & I_{s}
\end{array}\right] .
$$

Taking a certain model $\Theta$, all models with the same input-output behavior are given by

$$
\mathcal{I}_{\Theta}:=\left\{\bar{\Theta} \mid \bar{\Theta}=S_{\Theta}(T), \operatorname{det}(T) \neq 0\right\} .
$$

This set is called the indistinguishable set at $\Theta$, because it contains all the models that cannot be distinguished from $\Theta$ by looking at their input-output behavior. According to Lee and Poolla (1999), if the similarity map $S_{\Theta}$ is locally one-to-one at $I_{n}$, that is if $S_{\Theta}(T)=\Theta$ implies $T=I_{n}$, the connected component of $\mathcal{I}_{\Theta}$,

$$
\mathcal{I}_{\Theta}^{+}:=\left\{\bar{\Theta} \mid \bar{\Theta}=S_{\Theta}(T), \operatorname{det}(T)>0\right\},
$$

is a manifold. The tangent space of this manifold at the point $\Theta$ contains the directions along which a change in the parameters $\theta$ does not influence the value of the cost function $V_{N}(\theta, c, w)$. To improve the optimization algorithm, the update rule (5) should be changed such that the parameters $\theta$ are not changed along these directions. For this reason, we need to determine the tangent space of the manifold $\mathcal{I}_{\Theta}^{+}$.

In the sequel we assume that the similarity map is locally one-to-one. Lee and Poolla (1999) derived some, possibly conservative conditions on the system matrices that guarantee this. In the next lemma we reformulate their result for the model structure we consider in this paper.

Lemma 1 Let the observability matrix for $A_{i}$ and $C$ be given by

$$
\mathcal{O}_{n, i}:=\left[\begin{array}{c}
C \\
C A_{i} \\
C A_{i}^{2} \\
\vdots \\
C A_{i}^{n-1}
\end{array}\right] .
$$

If the matrix $\mathcal{O}_{n, i}$ has full rank for some $i \in\{1,2, \ldots, s\}$, then $S_{\Theta}(T)=\Theta$ implies $T=I_{n}$. 
Proof: If $\mathcal{O}_{n, i}$ has full rank, there exists a right pseudo-inverse $\mathcal{O}_{n, i}^{+}$such that $\mathcal{O}_{n, i}^{+} \mathcal{O}_{n, i}=I_{n}$. With the assumption $S_{\Theta}(T)=\Theta$ it follows that $\mathcal{O}_{n, i} T=\mathcal{O}_{n, i}$ and thus $T=\mathcal{O}_{n, i}^{+} \mathcal{O}_{n, i}=I_{n}$.

Note that a similar result can be proven by replacing the observability condition for $A_{i}$, $C$ with a controllability condition for $A_{i}$ and $B_{i}$.

To determine the tangent space of the manifold $\mathcal{I}_{\Theta}^{+}$, we linearize the similarity map $S_{\Theta}(T)$ :

$$
\begin{aligned}
S_{\Theta}(T) & \approx S_{\Theta}\left(I_{n}+T\right)-S_{\Theta}\left(I_{n}\right) \\
& \approx\left[\begin{array}{c}
\bar{A} \\
\bar{C}
\end{array}\right]\left[\begin{array}{ll}
T_{s} & 0_{n \times(m+1) s}
\end{array}\right]-\left[\begin{array}{c}
T \\
0_{l \times n}
\end{array}\right]\left[\begin{array}{ccc}
\bar{A} & \bar{B} & \bar{O}
\end{array}\right],
\end{aligned}
$$

where the last expression is obtained by approximating $\left(I_{n}+T\right)^{-1}$ by $I_{n}-T$ and neglecting all second order terms. By defining

$$
\Pi_{i}:=\left[\begin{array}{lll}
0_{n \times(i-1) n} & I_{n} & 0_{n \times(s-i) n}
\end{array}\right],
$$

it is possible to write (6) as

$$
S_{\Theta}(T) \approx \sum_{i=1}^{s}\left[\begin{array}{c}
\bar{A} \\
\bar{C}
\end{array}\right] \Pi_{i}^{T} T\left[\begin{array}{ll}
\Pi_{i} & 0_{n \times(m+1) s}
\end{array}\right]-\left[\begin{array}{c}
I_{n} \\
0_{l \times n}
\end{array}\right] T\left[\begin{array}{ccc}
\bar{A} & \bar{B} & \bar{O}
\end{array}\right] .
$$

Using the property $\operatorname{vec}(A B C)=\left(C^{T} \otimes A\right) \operatorname{vec}(B)$, we get

$$
\operatorname{vec}\left(S_{\Theta}(T)\right) \approx M_{\Theta} \operatorname{vec}(T)
$$

where

$$
M_{\Theta}:=\sum_{i=1}^{s}\left[\begin{array}{c}
\Pi_{i}^{T} \\
0_{(m+1) s \times n}
\end{array}\right] \otimes\left[\begin{array}{c}
\bar{A} \Pi_{i}^{T} \\
\bar{C} \Pi_{i}^{T}
\end{array}\right]-\left[\begin{array}{c}
\bar{A}^{T} \\
\bar{B}^{T} \\
\bar{O}^{T}
\end{array}\right] \otimes\left[\begin{array}{c}
I_{n} \\
0_{\ell \times n}
\end{array}\right]
$$

Therefore, the tangent space of the manifold $\mathcal{I}_{\Theta}^{+}$at the point $\Theta$ equals the column space of the matrix $M_{\Theta}$. Note that it is sufficient to look at $\Pi M_{\Theta}$, because the zero entries in $\Theta$ do not appear in $\theta$. Hence, if $\bar{\theta}=\Pi \operatorname{vec}\left(S_{\Theta}(T)\right)$, then

$$
\bar{\theta} \approx \Pi M_{\Theta} \operatorname{vec}(T) .
$$

Thus, the left null space of the matrix $\Pi M_{\Theta}$, which is the orthogonal complement of the tangent space of the manifold $\mathcal{I}_{\Theta}^{+}$at the point $\Theta$, contains the directions in which the parameters should be changed to obtain a change in the cost function $V_{N}(\theta, c, w)$. The next lemma shows that in general the matrix $M_{\Theta}$ has full rank.

Lemma 2 The similarity map $S_{\Theta}$ is locally one-to-one, that is $S_{\Theta}\left(I_{n}+T\right)=\Theta$ implies $T=0$, if and only if the matrix $M_{\Theta}$ has full rank.

Proof: The equality $S_{\Theta}\left(I_{n}+T\right)=\Theta$ is equivalent to

$$
\left[\begin{array}{c}
\bar{A} \\
\bar{C}
\end{array}\right]\left[\begin{array}{ll}
T_{s} & 0_{n \times(m+1) s}
\end{array}\right]-\left[\begin{array}{c}
T \\
0_{l \times n}
\end{array}\right]\left[\begin{array}{lll}
\bar{A} & \bar{B} & \bar{O}
\end{array}\right]=0 .
$$


With the definition of $M_{\Theta}$ this is in turn equivalent to

$$
M_{\Theta} \operatorname{vec}(T)=0 .
$$

It follows that $S_{\Theta}$ is locally one-to-one if and only if the matrix $M_{\Theta}$ has full rank, since in this case the only solution to (7) is $T=0$.

The left null space of the matrix $\Pi M_{\Theta}$ can be determined using a singular value decomposition. Given the singular value decomposition

$$
\Pi M_{\Theta}=\left[\begin{array}{ll}
U_{1}(\theta) & U_{2}(\theta)
\end{array}\right]\left[\begin{array}{c}
\Sigma(\theta) \\
0
\end{array}\right] V^{T}(\theta),
$$

every parameter vector $\theta$ can be decomposed into two parts: $\theta=U_{1}(\theta) U_{1}^{T}(\theta) \theta+U_{2}(\theta) U_{2}^{T}(\theta) \theta$, where the first part corresponds to the directions that do not influence the cost function, and the second part to the directions that change the value of the cost function. Based upon this observation, the update rule for the parameters (5) is changed such that the update is restricted to the directions that change the cost function;

$$
\left[\begin{array}{c}
\theta^{(i+1)} \\
c^{(i+1)} \\
w^{(i+1)}
\end{array}\right]=\left[\begin{array}{c}
\theta^{(i)} \\
c^{(i)} \\
w^{(i)}
\end{array}\right]-\mu^{(i)} \widetilde{H}_{N}\left(\lambda^{(i)}, \theta^{(i)}, c^{(i)}, w^{(i)}\right)^{-1} \widetilde{\Psi}_{N}^{T}\left(\theta^{(i)}, c^{(i)}, w^{(i)}\right) E_{N}\left(\theta^{(i)}, c^{(i)}, w^{(i)}\right),
$$

where

$$
\begin{aligned}
\widetilde{H}_{N}(\lambda, \theta, c, w) & :=U_{2}^{T}(\theta) \lambda I+\widetilde{\Psi}_{N}^{T}(\theta, c, w) \widetilde{\Psi}_{N}(\theta, c, w) \\
\widetilde{\Psi}_{N}(\theta, c, w) & :=\Psi_{N}(\theta, c, w) U_{2}(\theta) .
\end{aligned}
$$

Note that the matrix $U_{2}$ depends on the current parameters $\theta^{(i)}$, therefore the singular value decomposition (10) must be computed at each iteration. This is however, not so demanding compared to the computation of the gradient.

\section{Scheduling with the Input}

For the special case where the scheduling vector equals the input, $\phi_{k}=u_{k}$, the recurrent nature of the model (3)-(4) with respect to the weights is eliminated. The model is still recurrent in the state, but the weights $p_{i}$ do not depend on the state and can be computed independently. Once the weights have been computed, the state sequence follows by simulating a linear parameter-varying system, where the weights $p_{i}\left(u_{k}\right)$ are the time-varying parameters:

$$
x_{k+1}=A\left(p_{k}\right) x_{k}+B\left(p_{k}\right) u_{k}+O\left(p_{k}\right),
$$

with $p_{k}:=\left[p_{1}\left(u_{k}\right), p_{2}\left(u_{k}\right), \ldots, p_{s}\left(u_{k}\right)\right]$,

$$
A\left(p_{k}\right):=\sum_{i=1}^{s} A_{i} p_{i}\left(u_{k}\right)
$$

and similar definitions for $B\left(p_{k}\right)$ and $O\left(p_{k}\right)$. This greatly simplifies the gradient calculations, and hence the complexity of the training procedure. 
Gradient calculation for the identification of linear parameter-varying systems have been described by Lee and Poolla (1999). It appears that the gradient of $E_{N}(\theta, c, w)$ with respect to $\theta$ can be obtained by simulating a set of linear time-varying state equations. Note that to compute this gradient, we need to compute $\partial \widehat{y}_{k} / \partial \theta_{j}$ for every parameter $\theta_{j}$, and every time instant $k=1,2, \ldots, N$. We have

$$
\frac{\partial \widehat{y}_{k}}{\partial \theta_{j}}=C \mathcal{X}_{k}^{\theta_{j}}+\frac{\partial C}{\partial \theta_{j}} x_{k}
$$

with

$$
\mathcal{X}_{k}^{\theta_{j}}:=\frac{\partial x_{k}}{\partial \theta_{j}}
$$

which can be obtained for $k=1,2, \ldots, N$ by simulating the state equation

$$
\mathcal{X}_{k+1}^{\theta_{j}}=\sum_{i=1}^{s} p_{i}\left(u_{k}\right)\left(A_{i} \mathcal{X}_{k}^{\theta_{j}}+\frac{\partial A_{i}}{\partial \theta_{j}} x_{k}+\frac{\partial B_{i}}{\partial \theta_{j}} u_{k}+\frac{\partial O_{i}}{\partial \theta_{j}}\right),
$$

where $x_{k}$ is the state of (11).

The gradients with respect to the parameters $c$ and $w$ that describe the radial basis functions are also easy to compute. The gradient for the center $c_{j}$ of the $j$ th basis function is obtained from

$$
\frac{\partial \widehat{y}_{k}}{\partial c_{j}}=C \mathcal{X}_{k}^{c_{j}}
$$

where $\mathcal{X}_{k}^{c_{j}}$ is obtained by simulating

$$
\mathcal{X}_{k+1}^{c_{j}}=\sum_{i=1}^{s} p_{i}\left(u_{k}\right) A_{i} \mathcal{X}_{k}^{c_{j}}+\frac{\partial p_{i}\left(u_{k}\right)}{\partial c_{j}}\left(A_{i} x_{k}+B_{i} u_{k}+O_{i}\right),
$$

with

$$
\frac{\partial p_{i}\left(u_{k}\right)}{\partial c_{j}}= \begin{cases}\left(p_{j}\left(u_{k}\right)-p_{j}\left(u_{k}\right)^{2}\right) \operatorname{diag}(w)\left(u_{k}-c_{j}\right) & \text { for } i=j \\ -p_{i}\left(u_{k}\right) p_{j}\left(u_{k}\right) \operatorname{diag}(w)\left(u_{k}-c_{j}\right) & \text { for } i \neq j\end{cases}
$$

The expression for the gradient with respect to $w$ is similar.

For successful training, the dynamic equations of the model (3) and the ones involved in the gradient calculation, that is (13) and (14) have to be stable. Since $0<p_{i}\left(\phi_{k}\right)<1$, the stability of (3) depends on the matrices $A_{i}$. If the training is initiated with a boundedinput, bounded-output stable model, it is unlikely that the system matrices $A_{i}$ are modified towards instability, since the parameters $\theta$ are determined using a decent method that always decreases the cost function. If the matrices $A_{i}$ are such that the equation (3) is bounded-input, bounded-output stable, then the state sequence $x_{k}$ is bounded. From this it follows that the recursions (13) and (14) for calculating the gradients are also bounded-input, bounded-output stable.

The optimization based identification procedure described above, needs an initial estimate of the model parameters. Because the optimization problem is nonlinear and recurrent, the choice of this initial estimate can influence the outcome considerably. A natural way to 
initialize the model (3)-(4) is to estimate a global linear state space model and take all the local models equal to this linear model. Efficient subspace identification methods can be used for this (Verhaegen, 1994; Van Overschee and De Moor, 1996). Next, the radial basis functions are distributed uniformly over the range of the input $u_{k}$. In this way, the initial model equals the linear model and hence, the performance of the local linear models on the training data is always better than the performance of the global linear model. Using linear models for initialization has been proposed and motivated by Sjöberg (1997) and is also used by other authors, for example Gan and Danai (2000).

Note that training method outlined above, can easily be extended to deal with the case where $\phi_{k}=h\left(u_{k}, u_{k-1}, \ldots, u_{k-M}\right)$ for some known nonlinear map $h$ and integer $M$.

\section{Scheduling with the Input and Output}

A more general model is obtained if the scheduling vector is taken equal to

$$
\phi_{k}:=\left[\begin{array}{c}
u_{k} \\
C x_{k}
\end{array}\right]
$$

The reason for considering $C x_{k}$ is that the output $y_{k}$ is an estimate of this quantity. Actually, in the noise free case we have $y_{k}=C x_{k}$, hence we can take

$$
\phi_{k}:=\left[\begin{array}{l}
u_{k} \\
y_{k}
\end{array}\right]
$$

and the training procedure described in Section 4 can be applied. However, if there is noise, then this training procedure is not appropriate as illustrated by Nerrand et al. (1994); because $y_{k}-C x_{k}=v_{k} \neq 0$, there will be a difference between the training based on (15) and training based on (16). To analyze the difference between these two training methods, we fix the parameters $\theta, c$ and $w$, and derive the difference between the state sequences. The state sequence with the scheduling vector (15) is given by

$$
x_{k+1}=\sum_{i=1}^{s} p_{i}\left(u_{k}, C x_{k}\right)\left(A_{i} x_{k}+B_{i} u_{k}+O_{i}\right),
$$

and the state sequence obtained by using (16) instead, is given by

$$
\widetilde{x}_{k+1}=\sum_{i=1}^{s} p_{i}\left(u_{k}, y_{k}\right)\left(A_{i} \widetilde{x}_{k}+B_{i} u_{k}+O_{i}\right) .
$$

The state error $\varepsilon_{k}:=x_{k}-\widetilde{x}_{k}$ satisfies

$$
\varepsilon_{k+1}=\sum_{i=1}^{s} p_{i}\left(u_{k}, y_{k}\right) A_{i} \varepsilon_{k}+\mu_{k}
$$

with

$$
\mu_{k}=\sum_{i=1}^{s}\left(p_{i}\left(u_{k}, C x_{k}\right)-p_{i}\left(u_{k}, y_{k}\right)\right)\left(A_{i} x_{k}+B_{i} u_{k}+O_{i}\right)
$$


The state error $\varepsilon_{k}$ is bounded provided that the systems (17) and (18) are bounded-input, bounded-output stable. The state error will not go to zero as time increases, because the system (19) is driven by the signal $\mu_{k}$. The magnitude of $\mu_{k}$ depends on the difference between $p_{i}\left(u_{k}, C x_{k}\right)$ and $p_{i}\left(u_{k}, y_{k}\right)$ and thus on the noise level. Therefore, it is expected that a lower noise level yields a smaller state error $e_{k}$.

Note that the above analysis is for fixed parameters, since the parameters are changed during training, based on the value and gradient of the cost function, the estimated models obtained from the two procedures can differ considerably, and they frequently do in practice.

We can conclude that in the presence of noise, training with (16) is different from training with (15). Certain models can be very sensitive to the noise, so that even very small noise levels, like numerical errors arising from finite word lengths calculations, cause problems. The appropriate training method is the one based on (15). Unfortunately, this training procedure is not without problems.

For training based on (15), the gradient with respect to the parameters $\theta$ can again be computed by simulating a dynamic system, but now this system is no longer linear with respect to the state. The gradient can be computed from (12) with $\mathcal{X}_{k}^{\theta_{j}}$ satisfying

$$
\begin{gathered}
\mathcal{X}_{k+1}^{\theta_{j}}=\sum_{i=1}^{s} p_{i}\left(u_{k}, C x_{k}\right)\left(A_{i} \mathcal{X}_{k}^{\theta_{j}}+\frac{\partial A_{i}}{\partial \theta_{j}} x_{k}+\frac{\partial B_{i}}{\partial \theta_{j}} u_{k}+\frac{\partial O_{i}}{\partial \theta_{j}}\right) \\
+\sum_{i=1}^{s} \frac{\partial p_{i}\left(u_{k}, C x_{k}\right)}{\partial \theta_{j}}\left(A_{i} x_{k}+B_{i} u_{k}+O_{i}\right),
\end{gathered}
$$

Linearity is destroyed because $\partial p_{i}\left(u_{k}, C x_{k}\right) / \partial \theta_{j}$ depends on $\mathcal{X}_{k}^{\theta_{j}}$. Therefore, stability of the dynamic equation of the model (3) no longer implies stability of the dynamic equations involved in the gradient calculations. When the gradient calculations become unstable during training, the optimization algorithm breaks down.

Similar problems occur in training other types of nonlinear model structures, for example neural networks. In general, two training procedures can be distinguished. The first one, is the parallel method (Narendra and Parthasarathy, 1990), also called undirected training by Nerrand et al. (1994). In this method the model outputs are in a feed back loop and it is necessary to train a recurrent network. In the second method, called the series-parallel method (Narendra and Parthasarathy, 1990), or directed training (Nerrand et al., 1994), the recurrent nature is removed by replacing the feed back of the model outputs by the measured outputs. The parallel methods suffer from stability problems during training; therefore, series-parallel methods are often preferred. Although, with series-parallel methods the training procedure is well-behaved, the resulting models can have a bias (Nerrand et al., 1994) or might even give a completely different response if simulated in free-run.

As pointed out by Nerrand et al. (1994), a combination of parallel (undirected) and seriesparallel (directed) training is also possible, resulting in hybrid methods. In our discussion, training with (15) is a parallel method. Training with (16) is a hybrid method; the method is recurrent with respect to the state sequence, but not with respect to the scheduling variable. Similar arguments as given in Section 4 can be used to show that the hybrid method based on (16) does not suffer from stability problems during training and is therefore the preferred method. The drawback of this method is of course that the resulting methods might perform bad in free-run. 
Note that similar to the extension suggested at the end of Section 4 the identification procedure described above can easily be extended to deal with $\phi_{k}=h\left(y_{k}, y_{k-1}, \ldots, y_{k-L}, u_{k}, u_{k-1}, \ldots, u_{k-M}\right)$ for some known nonlinear map $h$ and integers $L$ and $M$.

\section{Dealing with Process Noise}

The presented training procedures for obtaining a model of the system (3)-(4) which is only disturbed by output measurement noise, can also be used to obtain a one-step-ahead predictor for the system

$$
\begin{aligned}
x_{k+1} & =\sum_{i=1}^{s} p_{i}\left(\phi_{k}\right)\left(A_{i} x_{k}+B_{i} u_{k}+O_{i}+K_{i} v_{k}\right) \\
y_{k} & =C x_{k}+v_{k},
\end{aligned}
$$

where a special kind of process noise acts on the state equation. It is easy to see that the one step-ahead predictor for this system is given by

$$
\begin{aligned}
x_{k+1} & =\sum_{i=1}^{s} p_{i}\left(\phi_{k}\right)\left(\left(A_{i}-K_{i} C\right) x_{k}+B_{i} u_{k}+O_{i}+K_{i} y_{k}\right) \\
\widehat{y}_{k} & =C x_{k} .
\end{aligned}
$$

This model can be trained in similar ways as discussed previously. The expressions for the gradient in the case $\phi_{k}=u_{k}$ are not difficult to derive, and again involve simulating a linear parameter-varying system. These expressions have been derived in the context of identification of linear parameter-varying systems in our paper: Verdult and Verhaegen 2000.

\section{Examples}

To demonstrate the usefulness of the proposed approach, we present in this section some examples that have been studied in the literature before. In all the examples, the local models are initialized using a linear state space model estimated by the PI-MOESP method described by Verhaegen (1994). The centers $c_{i}$ and widths $w_{i}$ of the weighting functions are initialized such that the weighting functions cover the operating region more or less uniformly. The scheduling variable $\phi_{k}$, the model order $n$, and the number of local linear models $s$, are determined by trial and error using the performance of the model on a fresh data set for decision. We are of opinion that in practice more systematic procedures are needed to determine these quantities, but such methods are outside the scope of this paper. In the examples, we use both the variance-accounted-for (VAF) and mean-square-error (MSE) to evaluate the performance of the models: the VAF is defined as

$$
\mathrm{VAF}=\max \left\{1-\frac{\operatorname{var}\left(y_{k}-\widehat{y}_{k}\right)}{\operatorname{var}\left(y_{k}\right)}, 0\right\} \times 100 \%,
$$

where $\widehat{y}_{k}$ denotes the output signal of the model, and $\operatorname{var}(\cdot)$ denotes the variance of a quasistationary signal; the MSE is simply $\operatorname{var}\left(y_{k}-\widehat{y}_{k}\right)$. 

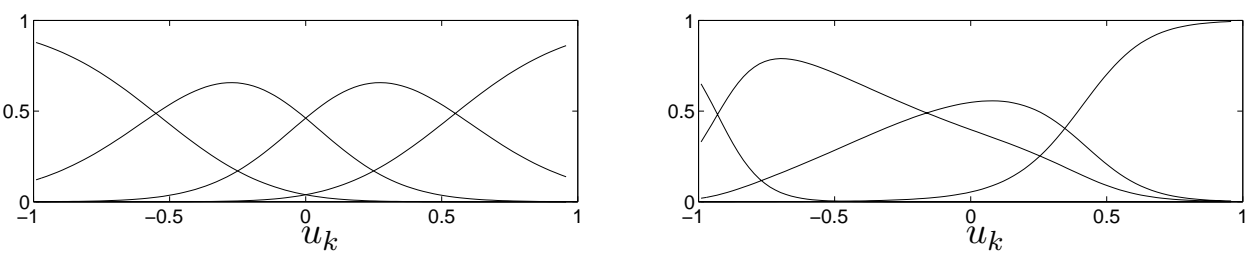

Figure 1: SISO example scheduled on the input: left, initial weights $p_{k}$ as a function of the input signal; right, weights $p_{k}$ after optimization.

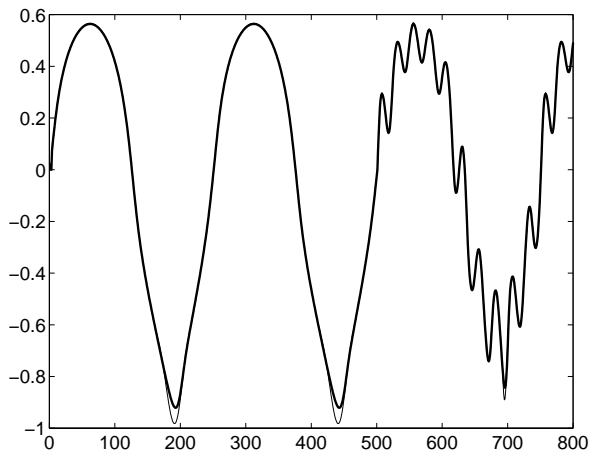

Figure 2: SISO example scheduled on the input: comparison between the output signal of the validation data (thick line) and the free-run simulation of the model (thin line). The lines are almost indistinguishable.

\subsection{SISO System Scheduled on the Input}

The first example system has been described by Narendra and Parthasarathy (1990) as an example for the use of neural networks to model dynamical systems. Nie (1994) has used this example to demonstrate the dynamic modeling capabilities of neural fuzzy networks and Boukhris et al. (1999) used it with local linear fuzzy models. The input-output description of the system is

$$
y_{k+1}=\frac{y_{k} y_{k-1} y_{k-2} u_{k-1}\left(y_{k-2}-1\right)+u_{k}}{1+\left(y_{k-2}\right)^{2}+\left(y_{k-1}\right)^{2}} .
$$

For identification a multi-step input signal is used; the steps in this signal have a fixed length of 10 samples and a random magnitude between -1 and 1 , determined by a uniform distribution. A data set of 800 samples is generated to train the local linear models. The initial weights $p_{k}$ and the optimized ones are shown in Figure 1. To asses the quality of the model a validation data set is generated using the input signal

$$
u_{k}= \begin{cases}\sin (2 \pi k / 250) & 1 \leq k \leq 500 \\ 0.8 \sin (2 \pi k / 250)+0.2 \sin (2 \pi k / 25) & 501 \leq k \leq 800 .\end{cases}
$$

The same validation data has been used by the previously mentioned authors. With four third order models scheduled on the input, the VAF on this validation data equals $99.9 \%$ and the MSE 0.0002. Figure 2 shows the outputs of the original system and of the model. 


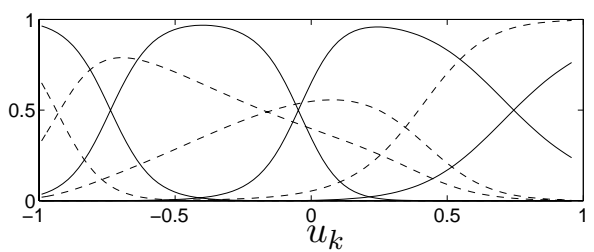

Figure 3: SISO example scheduled on the input, with output noise: weights $p_{k}$ after optimization (solid lines) compared with the optimized weights $p_{k}$ obtained from training without noise (broken lines).

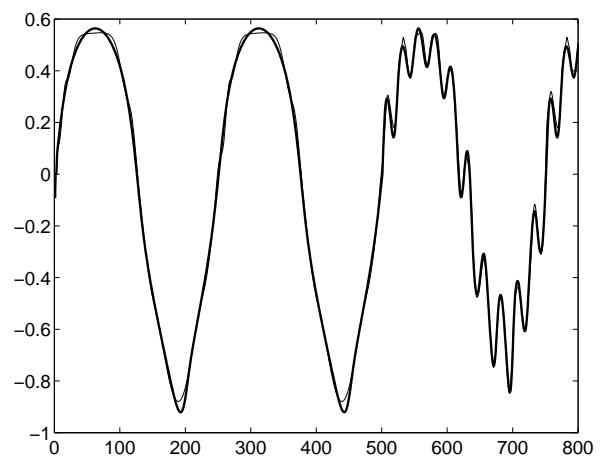

Figure 4: SISO example scheduled on the input, with output noise: comparison between the output signal of the validation data (thick line) and the free-run simulation of the model (thin line). The lines are almost indistinguishable.

Next, zero-mean white noise is added to the output signal, such that the VAF between the noise-free and noisy output equals $90.8 \%$ and the MSE 0.016. The training is influenced by the noise, as can be seen from Figure 3 which compares the weights after training with noisy data with the weights obtained by training with noise-free data. The weights clearly differ. However, the performance of the model on the validation data set is still very good: the VAF equals $99.8 \%$ and the MSE equals 0.0005 . Figure 4 shows the outputs of the original system and of the model obtained from noisy data. Note that the error on the validation data set is much smaller than the noise added to the identification data; therefore, the performance of the model is still satisfactory.

\subsection{SISO System Scheduled on the Output}

The following example system has also been described by Narendra and Parthasarathy (1990);

$$
y_{k+1}=\frac{y_{k} y_{k-1}\left(y_{k}+2.5\right)}{1+\left(y_{k-1}\right)^{2}+\left(y_{k}\right)^{2}}+u_{k} .
$$

Since, the input enters linearly, and the nonlinearity involves only the output, it is expected that the output should be used as a scheduling variable. To generate identification data, the system is excited with a random input signal, uniformly distributed between -1 and 1 . The 

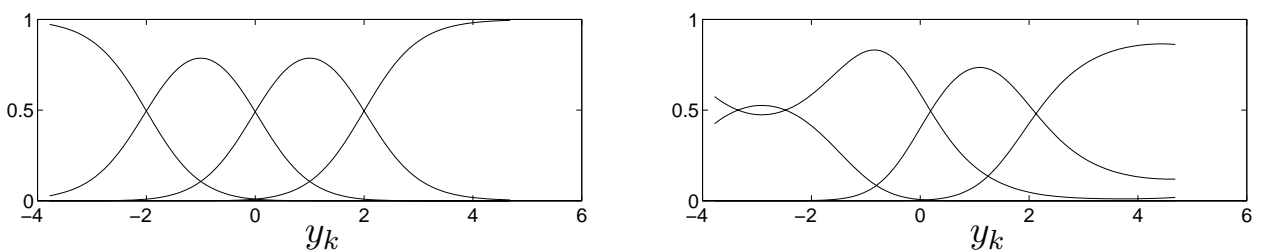

Figure 5: SISO example scheduled on the output: left, initial weights $p_{k}$ as a function of the output signal; right, weights $p_{k}$ after optimization.

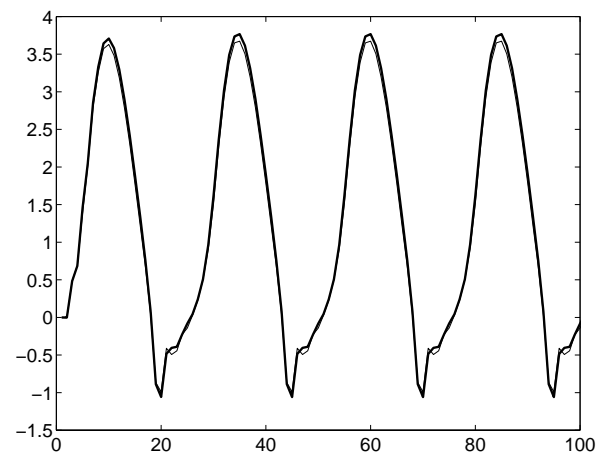

Figure 6: SISO example scheduled on the output: comparison between the output signal of the validation data (thick line) and the free-run simulation of the model (thin line). The lines are almost indistinguishable.

validation data set is generated with the input

$$
u_{k}=\sin (2 \pi k / 25),
$$

similar to Narendra and Parthasarathy (1990). With four second order models scheduled on the output, the VAF on this validation data set equals $99.8 \%$ and the MSE 0.01. A hybrid method based on scheduling with the measured output is used to train the models. Figure 5 shows the initial and optimized weights, and Figure 6 shows the outputs of the original system and of the model.

Zero-mean white noise is added to the output signal, such that the VAF between the noise-free and noisy output equals $90.3 \%$ and the MSE 0.24 . Figure 7 compares the weights after training with noisy data with the weights obtained by training with noise-free data. They clearly differ, so the training is influenced by the noise. The VAF on the validation data equals $98.8 \%$ and the MSE equals 0.066. Figure 8 shows the outputs of the original system and of the model obtained from noisy data. The difference between these outputs is now clearly visible, but note again that the error on the validation data is smaller than the noise added to the identification data.

\subsection{MIMO System}

The following MIMO example has been taken from Narendra and Parthasarathy (1992) and has also been studied by Nie (1994) and Boukhris et al. (1999). The system has two inputs 


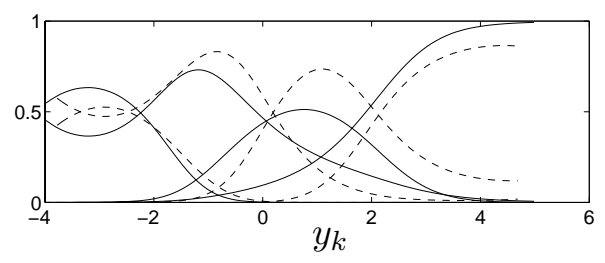

Figure 7: SISO example scheduled on the output, with output noise: weights $p_{k}$ after optimization (solid lines) compared with the optimized weights $p_{k}$ obtained from training without noise (broken lines).

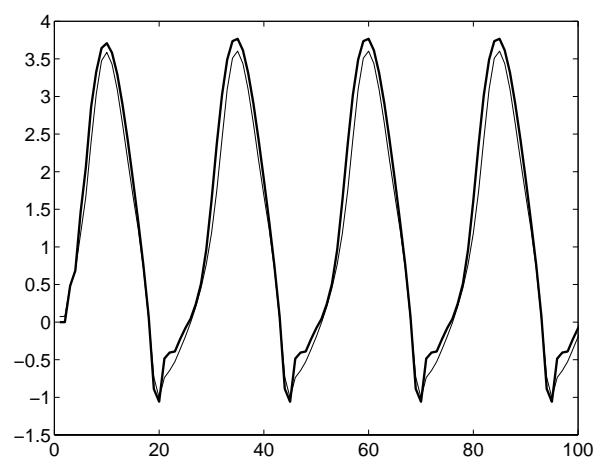

Figure 8: SISO example scheduled on the output, with output noise: comparison between the output signal of the validation data (thick line) and the free-run simulation of the model (thin line). 

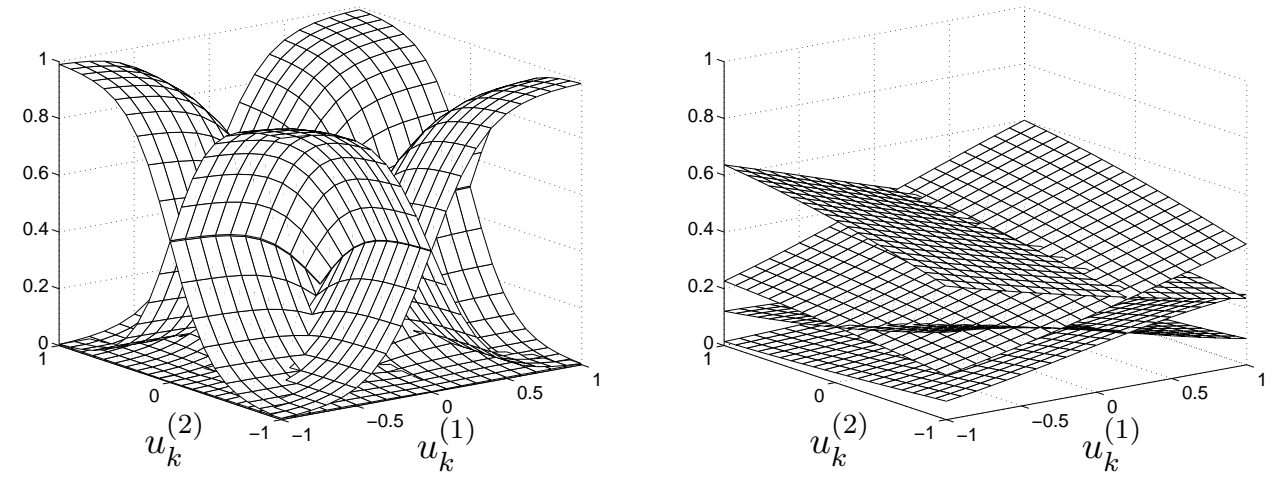

Figure 9: MIMO example: left, initial weights $p_{k}$ as a function of the two input signals; right, weights $p_{k}$ after optimization.

$u_{k}^{(1)}$ and $u_{k}^{(2)}$, and two outputs $y_{k}^{(1)}$ and $y_{k}^{(2)}$. The input-output description of the system is

$$
\begin{aligned}
& y_{k+1}^{(1)}=\frac{0.8\left(y_{k}^{(1)}\right)^{3}+\left(u_{k}^{(1)}\right)^{2} u_{k}^{(2)}}{2+\left(y_{k}^{(2)}\right)^{2}} \\
& y_{k+1}^{(2)}=\frac{y_{k}^{(1)}+y_{k}^{(1)} y_{k}^{(2)}+\left(u_{k-1}^{(1)}-0.5\right)\left(u_{k}^{(2)}+0.8\right)}{2+\left(y_{k}^{(2)}\right)^{2}} .
\end{aligned}
$$

The system is excited by taking the inputs equal to two independent random sequences of 1000 samples, uniformly distributed between -1 and 1 . The resulting data set is used to train the weights and local models of the system (3)-(4). An accurate description of the system is obtained by taking four third order models scheduled on the two inputs $u_{k}^{(1)}$ and $u_{k}^{(2)}$. The initial weights $p_{k}$ and the optimized ones are shown in Figure 9.

Following Narendra and Parthasarathy (1992) we evaluate the performance of the model using the input signals:

$$
\begin{aligned}
& u_{k}^{(1)}=\sin (2 \pi k / 250) \\
& u_{k}^{(2)}=\cos (2 \pi k / 250) .
\end{aligned}
$$

Figure 10 shows the response of the original system and of the model. The VAF on this validation data set is $97.9 \%$ for the first output and $99.2 \%$ for the second output; MSE for the outputs equals 0.0003 and 0.0016 , respectively. The performance of our local linear models is comparable to the performance of the fuzzy model described by Boukhris et al. (1999), but with a reduced model complexity. The performance is better than the neural network of Narendra and Parthasarathy (1992) and the fuzzy model of Nie (1994).

\subsection{Bioreactor}

Puskorius and Feldkamp (1994) have described a bioreactor benchmark problem for identification and control of nonlinear systems. This benchmark problem has been used for the identification of fuzzy local linear models by Cao et al. (1997). The benchmark involves a 

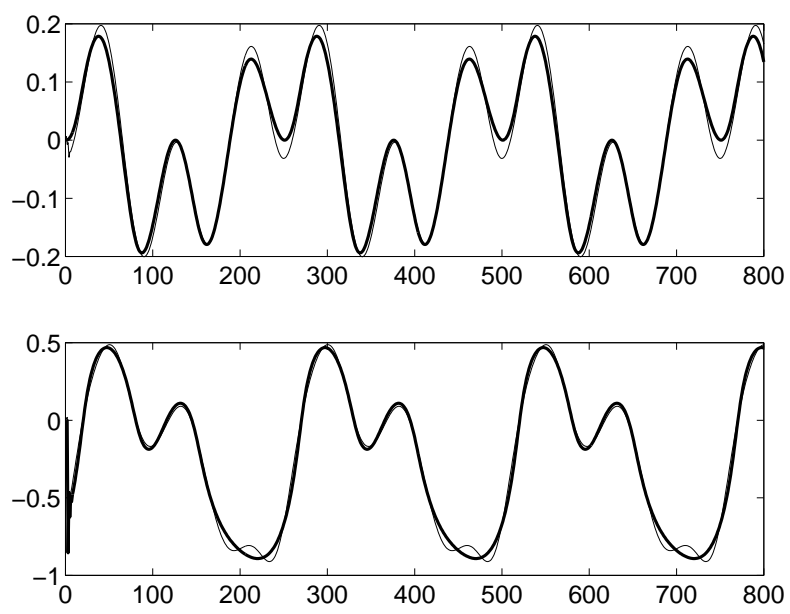

Figure 10: MIMO example: comparison between the two output signals of the validation data (thick lines) and the free-run simulation of the model (thin lines).

continuous flow stirred tank reactor that is described by two coupled nonlinear differential equations. These differential equations are integrated with a forward Euler scheme using a step size of $T=0.01$ time units; the resulting discrete-time equations are

$$
\begin{aligned}
& x_{k+1}^{(1)}=x_{k}^{(1)}+T\left(-x_{k}^{(1)} u_{k}+x_{k}^{(1)}\left(1-x_{k}^{(2)}\right)\right) \exp \left(\frac{x_{k}^{(2)}}{\Gamma}\right) \\
& x_{k+1}^{(2)}=x_{k}^{(2)}+T\left(-x_{k}^{(2)} u_{k}+x_{k}^{(1)}\left(1-x_{k}^{(2)}\right)\right) \exp \left(\frac{x_{k}^{(2)}}{\Gamma}\right) \frac{1+\beta}{1+\beta-x_{k}^{(2)}}
\end{aligned}
$$

where $\beta=0.02$ and $\Gamma=0.48$. The states $x_{k}^{(1)}$ and $x_{k}^{(2)}$ are dimensionless quantities describing the cell mass and the amount of nutrients in a constant volume tank. The input $u_{k}$ is the flow rate of nutrients into the tank, and equals the rate at which contents is removed from the tank. Only the first state is measurable, that is $y_{k}=x_{k}^{(1)}$.

The benchmark problem combines severe nonlinearity with a tendency to instability. However, we limit the modeling task to the stable region. We take the input signal equal to a multi-step signal with steps of 1000 time units with a random magnitude between 0 and 0.6 determined from a uniform distribution. The equations (20)-(21) are used to generate 50000 data samples. The resulting input and output data sets are resampled at $1 / 50$, so 1000 data samples are available for identification. The same resampling is used by Puskorius and Feldkamp (1994).

First, we tried to schedule on the output. With two first order models a good performance was obtained if the real output, so not the model output, is used as a scheduling variable. However, it is desirable to simulate the model in free-run, that is with the model output as scheduling variable. In this case the models performs very poorly. A possible explanation is that small errors in the output signal result in wrong scheduling and thus the model output diverges from the real output.

Next, we scheduled on the input. It turned out that three third order models scheduled on $u_{k}$ and $u_{k-1}$ give a satisfactory performance. On a fresh data set, the VAF equals $99.1 \%$ 

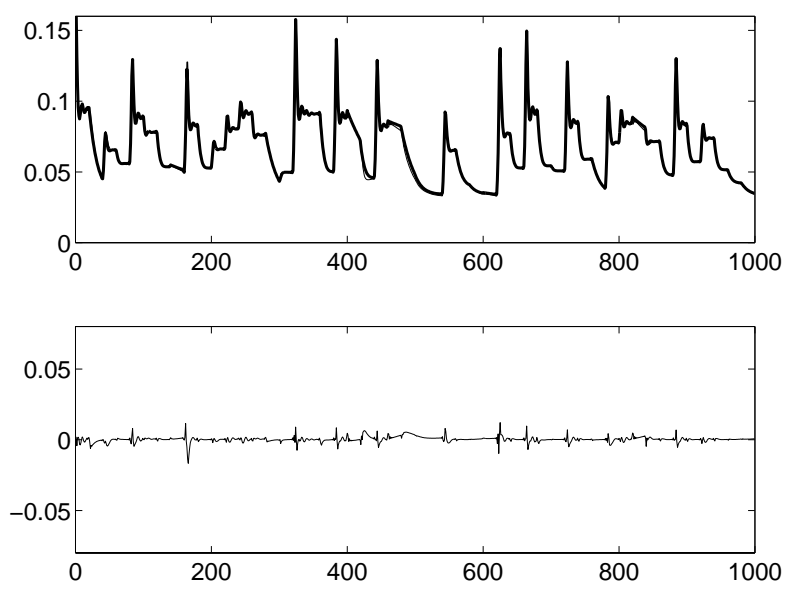

Figure 11: Bioreactor example: comparison between the validation data and the free-run simulation of the local linear models; The top figure shows the original output signal (thick line) and the model output (thin line); these signals are almost indistinguishable. The bottom figure shows the error between validation data and model output.

and the MSE $4 \cdot 10^{-6}$. Figure 11 shows the real output of this validation data set together with the model output. For comparison, Figure 12 shows the output of the linear model that was used to initialize the local models. The initial weights $p_{k}$ and the optimized ones are shown in Figure 13.

\section{Conclusions}

We described a novel identification method for local linear state space models scheduled by the input or a combination of the input and output. The method does not require full state measurement, and can deal with measurement noise and certain types of process noise in a consistent way. Normalized radial basis functions are used as weighting functions that combine the local state space models to obtain a global description of the input-output behavior. The system matrices of the local models are fully parametrized. An iterative projected gradient search algorithm has been described to identify the local models and the centers and widths of the radial basis functions. The projected gradient method deals with the nonuniqueness of the fully parametrized state space representation by first calculating at every iteration the direction in which a change of parameters does not influence the input-output behavior of the model, and subsequently projecting these directions out of the parameter update.

Two special cases of scheduling were discussed. When the scheduling is based only on the input signals, stability of the dynamic gradient calculations can be guaranteed, and hence the training is well-behaved. In the more general case where scheduling is also based on the output signals, two training methods are possible, each having their own advantages and disadvantages. One training method is based on scheduling with the measured outputs; this method behaves well during training, but the resulting model can perform poorly when simulated in free-run. The other training method that uses the model output for scheduling, does not have this problem, but can suffer from stability problems during training. 

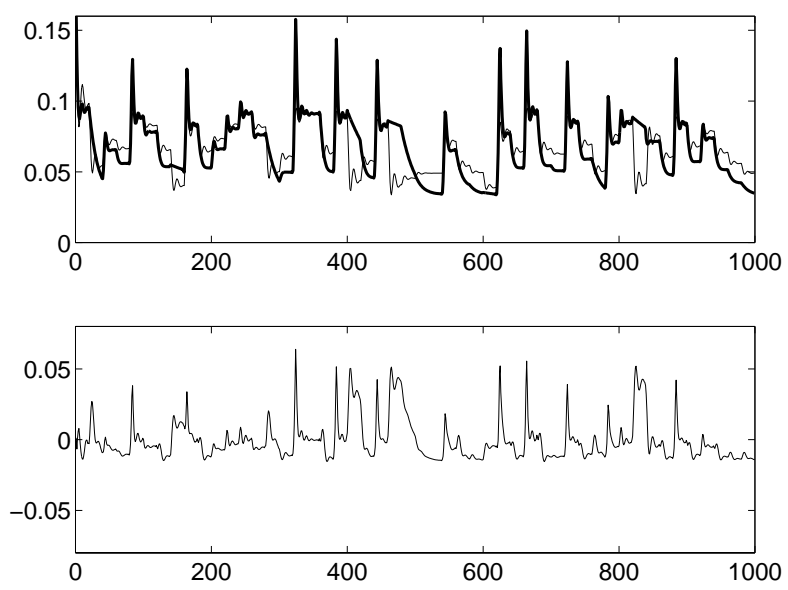

Figure 12: Bioreactor example: comparison between the validation data and the free-run simulation of the linear time-invariant model; The top figure shows the original output signal (thick line) and the model output (thin line). The bottom figure shows the error between validation data and model output.
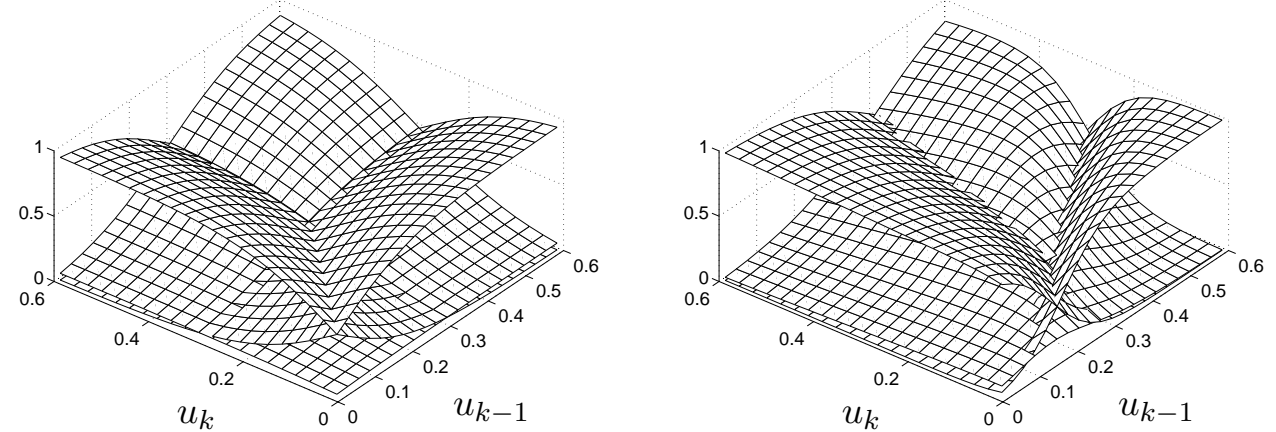

Figure 13: Bioreactor example: left, initial weights $p_{k}$ as a function of $u_{k}$ and $u_{k-1}$; right, weights $p_{k}$ after optimization. 
To initialize the training procedure, we suggest to estimate a global linear model and use this model to initialize all the local models, and in addition distribute the weighting functions uniformly over the operating range of the scheduling vector. With this initialization, the performance of the local linear models on the identification data will always be better than that of the global linear model.

Several examples that have been studied in the literature before, have been used to demonstrate the potential of the proposed methods.

\section{Acknowledgements}

This research is supported by the Dutch Technology Foundation (STW) under project number DEL55.3891.

\section{References}

Bottou, Léon and Vladimir Vapnik (1992). Local learning algorithms. Neural Computation 4(6), 888-900.

Boukhris, Anass, Gilles Mourot and José Ragot (1999). Non-linear dynamic system identification: A multi-model approach. International Journal of Control 72(7/8), 591-604.

Cao, S. G., N. W. Rees and G. Feng (1997). Analysis and design for a class of complex control systems part I: Fuzzy modelling and identification. Automatica 33(6), 1017-1028.

Gan, Chengyu and Kourosh Danai (2000). Model-based recurrent neural network for modeling nonlinear dynamic systems. IEEE Transactions on Systems, Man, and Cybernetics - PartB: Cybernetics 30(2), 344-351.

Haykin, Simon (1999). Neural Networks: A Comprehensive Foundation (second ed.). Upper Saddle River, New Jersey: Prentice Hall. ISBN 0-13-273350-1.

Johansen, Tor Arne (1997). On Tikhonov regularization, bias and variance in nonlinear system identification. Automatica 33(3), 441-446.

Johansen, Tor Arne, Robert Shorten and Roderick Murray-Smith (2000). On the interpretation and identification of dynamic Takagi-Sugeno fuzzy models. IEEE Transactions on Fuzzy Systems 8(3), 297-313.

Lee, Lawton H. and Kameshwar Poolla (1999). Identification of linear parameter-varying systems using nonlinear programming. Journal of Dynamic Systems, Measurement and Control 121(1), 71-78.

McKelvey, Tomas and Anders Helmersson (1997). System identification using an overparametrized model class: Improving the optimization algorithm. In Proceedings of the 36th IEEE Conference on Decision and Control, San Diego, California (December), pp. 2984-2989.

Murray-Smith, Roderick and Tor Arne Johansen (1997a). Local learning in local model networks. See Murray-Smith and Johansen (1997b), Chapter 7, pp. 185-210. 
Murray-Smith, Roderick and Tor Arne Johansen (Eds.) (1997b). Multiple Model Approaches to Modelling and Control. London: Taylor \& Francis. ISBN 0-7484-0595-X.

Narendra, Kumpati S. and Kannan Parthasarathy (1990). Identification and control of dynamical systems using neural networks. IEEE Transactions on Neural Networks 1(1), 4-27.

Narendra, Kumpati S. and Kannan Parthasarathy (1992). Neural networks and dynamical systems. International Journal of Approximate Reasoning 6, 109-131.

Nerrand, Olivier, Pierre Roussel-Ragot, Léon Personnaz and Gérard Dreyfus (1993). Neural networks and non-linear adaptive filtering: Unifying concepts and new algorithms. Neural Computation 5(2), 165-197.

Nerrand, Olivier, Pierre Roussel-Ragot, Dominique Urbani, Léon Personnaz and Gérard Dreyfus (1994). Training recurrent neural networks: Why and how? An illustration in dynamical process modeling. IEEE Transactions on Neural Networks 5(2), 178-184.

Nie, Junhong (1994). A neural approach to fuzzy modeling. In Proceedings of the 1994 American Control Conference, Baltimore, Maryland (June), pp. 2139-2143.

Puskorius, Gintaras V. and Lee A. Feldkamp (1994). Neurocontrol of nonlinear dynamical systems with Kalman filter trained recurrent networks. IEEE Transactions on Neural Networks 5(2), 279-297.

Rivals, Isabelle and Léon Personnaz (1996). Black-box modeling with state-space neural networks. In R. Zbikowski and K. J. Hunt (Eds.), Neural and Adaptive Control Technology, pp. 237-264. Singapore: World Scientific. ISBN 981-02-2557-1.

Shorten, Robert and Roderick Murray-Smith (1997). Side-effects of normalising basis functions in local model networks. See Murray-Smith and Johansen (1997b), Chapter 8, pp. $211-228$.

Shorten, Robert, Roderick Murray-Smith, Roger Bjørgan and Henrik Gollee (1999). On the interpretation of local models in blended multiple model structures. International Journal of Control 72(7/8), 620-628.

Sjöberg, Jonas (1997). On estimation of nonlinear black-box models: How to obtain a good initialization. In Proceedings of the 1997 IEEE Workshop Neural Networks for Signal Processing VII, Amelia Island Plantation, Florida (September), pp. 72-81.

Sjöberg, Jonas and Lennart Ljung (1995). Overtraining, regularization and searching for a minimum, with applications to neural networks. International Journal of Control 62(6), $1391-1407$.

Sjöberg, Jonas, Qinghua Zhang, Lennart Ljung, Albert Benveniste, Bernard Delyon, PierreYves Glorennec, Håkan Hjalmarsson and Anatoli Juditsky (1995). Nonlinear black-box modeling in system identification: A unified overview. Automatica 31(12), 1691-1724.

Skeppstedt, Anders, Lennart Ljung and Mille Millnert (1992). Construction of composite models from observed data. International Journal of Control 55(1), 141-152. 
Stark, J. (1999). Delay embeddings for forced systems part I: Deterministic forcing. Journal of Nonlinear Science 9, 255-332.

Stark, J., D. S. Broomhead, M. E. Davies and J. Huke (1997). Takens embedding theorems for forced and stochastic systems. Nonlinear Analysis, Theory, Methods $\& 5$ Applications 30(8), $5303-5314$.

Van Overschee, Peter and Bart De Moor (1996). Subspace Identification for Linear Systems; Theory, Implementation, Applications. Dordrecht, The Netherlands: Kluwer Academic Publishers. ISBN 0-7923-9717-7.

Verdult, Vincent and Michel Verhaegen (2000). Identification of multivariable LPV state space systems by local gradient search. Technical Report UT/TN/SCE-2000.016, Faculty of Applied Physics, University of Twente, Enschede, The Netherlands. To be presented at the European Control Conference 2001, Porto, Portugal, September 2001.

Verhaegen, Michel (1994). Identification of the deterministic part of MIMO state space models given in innovations form from input-output data. Automatica 30(1), 61-74. 